\title{
Sodium Picosulfate/Magnesium Oxide/Citric Acid Laxative
}

National Cancer Institute

\section{Source}

National Cancer Institute. Sodium Picosulfate/Magnesium Oxide/Citric Acid Laxative. NCI

Thesaurus. Code C80015.

An oral laxative formulation containing the stimulant cathartic sodium picosulfate as the primary active ing redient. Picosulfate acts on the enteric nerves in the intestinal wall to increase muscle contractions, thereby stimulating peristaltic action and promoting defecation. Other active ingredients are osmotic agents that increase stool water content. 